Annales Academiæ Scientiarum Fennicæ

Series A. I. Mathematica

Volumen 14, 1989, 47-55

\title{
SETS OF ZERO ELLIPTIC HARMONIC MEASURES
}

\section{O. Martio}

\section{Introduction}

An elliptic partial differential equation $\nabla \cdot A(x, \nabla u(x))=0$ in a domain $G$ with $|A(x, h)| \approx|h|^{p-1}$ produces a solution $\omega$ called an $A$-harmonic measure. For $p \neq 2, \omega$ is non-additive and hence does not define a measure in the Borel sets of $\partial G$ as the classical harmonic measure induced by the Laplace operator $A(x, h)=h$ does. The most interesting problem associated with $\omega$ is to determine the class of subsets $E$ of $\partial G$ such that $\omega(E)=0$. This class depends on $A$. For example, in the plane unit disk $B$ there is a linear elliptic operator $A(x, h) \approx h$ which induces $\omega$ such that $\omega(E)>0$ for some compact set $E \subset \partial B$ whose linear measure is zero. Such an operator $A$ can be constructed using quasiconformal mappings, see [GLM 2] and [CFK]. Hence $\omega$ essentially differs from the ordinary plane harmonic measure induced by the Laplace operator. Contrary to this example we show in this paper that there exists a reasonable class of subsets $E$ of $\partial G$ such that $\omega(E)=$ 0 for all operators $A$. Clearly $\partial G$ must be sufficiently thick for this purpose. For compact subsets $E$ of $\partial G$ our main result, Theorem 3.1, is formulated in terms of certain metric conditions of $E$ with respect to $\partial G$. Here the quasihyperbolic distance [GP] is useful. Surprisingly, for $G=B$, the unit ball of $R^{n}$, Theorem 3.1 shows that there are compact sets $E \subset \partial B$ whose Hausdorff dimension is arbitrary near $n-1$ and $\omega(E)=0$ for all $A$. By the above example this condition cannot be replaced by the condition that the $(n-1)$-dimensional Hausdorff measure of $E$ is $=0$.

For $p=n$ these problems were first studied in [GLM 2] and [HM]. Conditions for $\omega(E)>0$ were given in [GLM 2,4.10] and [M]. If $\partial G$ is "thick", then these results can be used to prove the counterpart of B. Øksendal's theorem for the $A$-harmonic measures $\omega$, see [HM, Theorem 4.1] and [H, Theorem A]. Our main theorem, Theorem 3.1, can also be used to study sets $E$ in $\partial G$ which cannot be seen easily from $G$. We say that such sets $E$ are buried in $\partial G$ and prove that $\omega(E)=0$ for all $A$; this result slightly generalizes [H, Theorem A]. Using stochastic methods $\varnothing \mathrm{ksendal}[\varnothing]$ has also studied the corresponding problems for $p=2$ and for linear operators $A$.

Suppose that $G$ is a bounded domain in $R^{n}$ and that $1<p \leq n$. We shall study partial differential operators $A: G \times R^{n} \rightarrow R^{n}$ which satisfy the following assumptions: 
a) For each $\varepsilon>0$ there exists a compact subset $F$ of $G$ such that $A \mid F \times R^{n}$ is continuous and $m(G \backslash F)<\varepsilon$.

b) There exist positive constants $\gamma_{1}$ and $\gamma_{2}$ such that for a.e. $x \in G$

$$
\begin{gathered}
|A(x, h)| \leq \gamma_{1}|h|^{p-1}, \\
A(x, h) \cdot h \geq \gamma_{2}|h|^{p}
\end{gathered}
$$

for all $h \in R^{n}$.

c) For a.e. $x \in G$

$$
\left(A\left(x, h_{1}\right)-A\left(x, h_{2}\right)\right) \cdot\left(h_{1}-h_{2}\right)>0, \quad h_{1} \neq h_{2} .
$$

d) For a.e. $x \in G$

$$
A(x, \lambda h)=|\lambda|^{p-2} \lambda A(x, h)
$$

for $\lambda \in R \backslash\{0\}$ and $h \in R^{n}$.

A continuous function $u: G \rightarrow R$ is a solution of the equation

$$
\nabla \cdot A(x, \nabla u(x))=0
$$

if $u$ belongs to the Sobolev space $\operatorname{loc} W_{p}^{1}(G)$, i.e., $u$ is ACL ${ }^{p}$, and if

$$
\int_{G} A(x, \nabla u(x)) \cdot \nabla \phi(x) d m(x)=0
$$

for all $\phi \in C_{0}^{\infty}(G)$. We call solutions of (1.3) $A$-harmonic. A lower semicontinuous function $u: G \rightarrow R \cup\{\infty\}$ is $A$-superharmonic if it satisfies the $A$-comparison principle, i.e., if for every domain $D \subset \subset G$ and every $A$-harmonic function $h \in$ $C(\bar{D})$ in $D, h \leq u$ in $\partial D$ implies $h \leq u$ in $D$. These functions form a similar, but in general non-linear, potential theory as ordinary harmonic and superharmonic functions do, see [GLM 1] and [HK].

Finally, let $E$ be a subset of $\partial G$. The upper class $\mathcal{U}$ consists of all $A$ superharmonic functions $u: G \rightarrow R \cup\{\infty\}$ such that

$$
\liminf _{x \rightarrow y} u(x) \geq \chi_{E}(y)
$$

for each $y \in \partial G$. Here $\chi_{E}$ is the characteristic function of $E$. It can be shown that

$$
\omega(E, G ; A)(x)=\inf _{u \in \mathcal{U}} u(x), \quad x \in G,
$$

defines an $A$-harmonic function $\omega=\omega(E, G ; A)$, called the $A$-harmonic measure of $E$ with respect to $G$. For this construction see [HK] and [GLM 2]. The set $E$ has zero $A$-harmonic measure, if $\omega(x)=0$ for some $x \in G$, or equivalently $\omega(x)=0$ for all $x \in G$. The last assertation follows from Harnack's inequality, see Lemma 3.3 below. In this case we simply write $\omega=0$. 


\section{Sets of $A$-harmonic measure zero}

Let $G$ be a bounded domain in $R^{n}$. We assume that $G$ is $A$-Dirichlet regular, i.e., for each $\psi \in C(\partial G)$ there is a (unique) function $u \in C(\bar{G})$ such that $u$ is $A$-harmonic in $G$ and that $u \mid \partial G=\psi$. The function $u$ is called the $A$-harmonic function with boundary values $\psi$. The following lemma is a generalization of [GLM 2, 4.9].

2.1. Lemma. Suppose that $E$ is a compact subset of $\partial G$. Let $\omega=$ $\omega(E, G ; A)$. Then $\omega=0$ if and only if there is $c \in[0,1)$ and a sequence of neighborhoods $\mathcal{U}_{i}, i=1,2, \ldots$, of $E$ such that

$$
\bigcap \mathcal{U}_{i} \cap G=\emptyset
$$

and

$$
\omega(x) \leq c \quad \text { for each } x \in G \cap \partial \mathcal{U}_{i}, \quad i=1,2, \ldots
$$

Proof. For the only if part choose $c=0$ and $\mathcal{U}_{i}=E+B(1 / i), i=1,2, \ldots$. Here $B(r)$ denotes the open ball of radius $r>0$ centered at 0 .

For the converse part we first show that

$$
u(x) \leq c
$$

for each $x \in G$. Fix $x \in G$. By (a) there is $\mathcal{U}_{i}$ such that $x \notin \mathcal{U}_{i}$. If $x \in \partial \mathcal{U}_{i}$, then (2.2) follows from (b). Assume that $x \in G \backslash \overline{\mathcal{U}}_{i}$. Let $V$ be the $x$-component of $G \backslash \overline{\mathcal{U}}_{i}$. Let $y \in \partial V$. If $y \in G$, then $y \in \partial \mathcal{U}_{i}$ and hence $\omega(y) \leq c$ by (b). If $y \notin G$, then let $\psi \in C(\partial G)$ be such that $\psi(y)=0, \psi \mid E=1$ and $0 \leq \psi \leq 1$. Let $u$ be the $A$-harmonic function with boundary values $\psi$. Then $u(y)=0$ and since $u$ belongs to the upper class $\mathcal{U}, \omega \leq u$ in $G$. Hence we obtain

$$
\lim _{z \rightarrow y} \omega(z)=0 \text {. }
$$

Thus in both cases

$$
\limsup _{z \rightarrow y} \omega(z) \leq c
$$

and this holds for every $y \in \partial V$. Now constants are $A$-harmonic functions, hence the $A$-comparison principle yields $\omega \leq c$ in $V$ and we have shown $\omega(x) \leq c$ as required.

Next we complete the proof for the converse part. If $c=0$, then $\omega=0$ as required. If $c>0$ and $\omega \neq 0$, then $\omega>0$ and hence

$$
\omega<\omega / c \quad \text { in } G \text {. }
$$

On the other hand, $\omega / c \leq 1$ in $G$ by (2.2) and if $u$ belongs to the upper class $\mathcal{U}$ for $\omega$, then

$$
\omega / c \leq u
$$

by the $A$-comparison principle. Note that $\lim _{z \rightarrow y} \omega(z)=0$ for every $y \in \partial G \backslash E$; this can be proved as (2.3). By $(2.5), \omega / c \leq \omega$ and hence we obtain a contradiction from (2.4). This completes the proof. 


\section{Quasihyperbolic distance and $A$-harmonic measure}

Let $E$ be a closed set in $R^{n}$ and $D=R^{n} \backslash E$. If $x_{1}, x_{2} \in D$, then the quasihyperbolic distance $k_{D}\left(x_{1}, x_{2}\right)$ of $x_{1}$ and $x_{2}$ is

$$
k_{D}\left(x_{1}, x_{2}\right)=\inf _{\gamma} \int_{\gamma} d(x, E)^{-1} d s
$$

where the infimum is taken over all rectifiable curves $\gamma$ joining $x_{1}$ and $x_{2}$ in $D$. Here $d(x, E)$ denotes the distance from $x$ to $E$. If no such curves exist, i.e., if $x_{1}$ and $x_{2}$ belong to different components of $D$, then we set $k_{D}\left(x_{1}, x_{2}\right)=\infty$.

Let $G$ be a domain in $R^{n}$. We say that $G$ satisfies a $p$-capacity density condition if for some $c_{0}>0$ and $r_{0}>0$

$$
\operatorname{cap}_{p}(\bar{B}(x, r) \cap \mathcal{C} G, B(x, 2 r)) \geq c_{0} r^{n-p}
$$

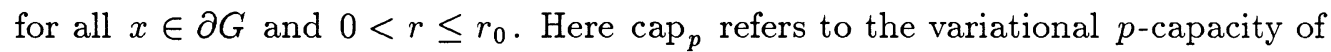
the condenser $E=(\bar{B}(x, r) \cap \mathcal{C} G, B(x, 2 r))$, i.e.,

$$
\operatorname{cap}_{p} E=\inf \int_{B(x, 2 r)}|\nabla u|^{p} d m
$$

where the infimum is taken over all functions $u \in C_{0}^{\infty}(B(x, 2 r))$ such that $u \geq 1$ in $\bar{B}(x, r) \cap \mathcal{C} G$.

3.1. Theorem. Let $G$ be a bounded domain satisfying a $p$-capacity density condition. Suppose that $E$ is a compact subset of $\partial G$ such that there exist a sequence of neighborhoods $\mathcal{U}_{i}, i=1,2, \ldots$, of $E$ and $M<\infty$ with

(a) $\cap \mathcal{U}_{i} \cap G=\emptyset$ and

(b) for each $i=1,2, \ldots$ and $x \in \partial \mathcal{U}_{i} \cap G$ there is $y \in \partial G$ with $k_{D}(x, y) \leq M$, $D=R^{n} \backslash E$.

Then $\omega(E, G ; A)=0$.

The proof is based on two lemmas. The first is essentially due to V.G. Maz'ya [Maz]. We shall employ the short argument due to Heinonen [H, Lemma 5.2].

3.2. Lemma. Let $F$ be a closed set in a ball $B\left(x_{0}, 2 r\right)$. If $u$ is a continuous function in $B\left(x_{0}, 2 r\right)$ such that $u \mid F=1,0 \leq u \leq 1$ and $u$ is a solution of (1.3) in $B\left(x_{0}, 2 r\right) \backslash F$, then

$$
u(x) \geq c_{1} r^{(p-n) /(p-1)} \operatorname{cap}_{p}\left(F \cap \bar{B}\left(x_{0}, r\right), B\left(x_{0}, 2 r\right)\right)^{1 /(p-1)}
$$

for each $x \in B\left(x_{0}, r\right)$. Here the constant $c_{1}$ depends only on $\gamma_{1}, \gamma_{2}, p$ and $n$. 

$5.2]$

Proof. Let $\omega=\omega\left(F \cap \bar{B}\left(x_{0}, r\right), B\left(x_{0}, 2 r\right) \backslash\left(F \cap \bar{B}^{n}\left(x_{0}, r\right)\right) ; A\right)$. By [H, Lemma

$$
\omega(x) \geq c_{1} r^{(p-n) /(p-1)} \operatorname{cap}_{p}\left(F \cap \bar{B}^{n}\left(x_{0}, r\right), B\left(x_{0}, 2 r\right)\right)^{1 /(p-1)}
$$

for each $x \in B\left(x_{0}, r\right) \backslash F$ and $c_{1}>0$ depends only on $\gamma_{1}, \gamma_{2}, p$ and $n$. Next fix $x \in B\left(x_{0}, r\right) \backslash F$ and let $V$ be the $x$-component of $B\left(x_{0}, 2 r\right) \backslash F$. Now $\liminf u(z) \geq \lim \sup \omega(z)$ as $z$ approaches $y \in \partial V$ in $V$; note that $0 \leq \omega \leq 1$ and that $\lim _{z \rightarrow y} \omega(z)=0$ for all $y \in \partial B\left(x_{0}, 2 r\right)$ because balls are always $A$ Dirichlet regular. Hence by the $A$-comparison principle $u \geq \omega$ in $V$ and thus the required inequality follows from the corresponding inequality for $\omega$. 269].

The next lemma is the well known Harnack inequality, see e.g. [S, pp. 264-

3.3. Lemma. Let $u$ be a non-negative solution of (1.3) in $B\left(x_{0}, 2 r\right)$. Then

$$
\sup _{x \in B\left(x_{0}, r\right)} u(x) \leq c_{2} \inf _{x \in B\left(x_{0}, r\right)} u(x)
$$

where the constant $c_{2}$ depends only on $\gamma_{1}, \gamma_{2}, p$ and $n$.

Proof for Theorem 3.1. Since $G$ is bounded, we may assume that the inequality in the $p$-capacity density condition holds for all $r \in(0, \operatorname{diam} G)$. Write $\omega=\omega(E, G ; A)$. We shall show that there is $c \in[0,1)$ such that

$$
\omega(x) \leq c
$$

for all $x \in \partial \mathcal{U}_{i} \cap G, i=1,2, \ldots$ Lemma 2.1 then completes the proof. Observe that since $G$ satisfies a $p$-capacity density condition, $G$ is $A$-Dirichlet regular, see [Maz]. This implies that $\lim _{x \rightarrow y} \omega(x)=0$ for all $y \in \partial G \backslash E$.

Fix $i=1,2, \ldots$ and let $x \in \partial \mathcal{U}_{i} \cap G$. Choose $y \in \partial G$ with $k_{D}(x, y) \leq M$. Let $\gamma$ be a rectifiable curve in $D$ joining $x$ to $y$ with

$$
\int_{\gamma} d(z, E)^{-1} d s \leq M+1 \text {. }
$$

Next choose points $z_{1}, \ldots, z_{j}$ and radii $r_{1}, \ldots, r_{j}$ inductively as follows. Set $z_{1}=x$ and $r_{1}=d\left(z_{1}, E\right) / 4$. Assume that $z_{1}, \ldots, z_{i}$ have been chosen and let $\gamma_{i}$ denote the part of $\gamma$ from $z_{i}$ to $y$. If $\partial G \cap \bar{B}\left(z_{i}, 2 r_{i}\right) \neq \emptyset$, then we set $j=i$ and end the process. If $\partial G \cap \bar{B}\left(z_{i}, 2 r_{i}\right)=\emptyset$, then choose $z_{i+1}$ to be the last point where $\gamma_{i}$ meets $\partial B\left(z_{i}, r_{i}\right)$ and put $r_{i+1}=d\left(z_{i+1}, E\right) / 4$. Since $y \in \partial G \backslash E$, this process ends after a finite number of steps.

Next we obtain an upper bound for $j$ in terms of $M$. Fix $i=1, \ldots, j-1$ and let $\gamma_{i}$ be the part of $\gamma$ from $z_{i}$ to $z_{i+1}$. Pick $z^{\prime} \in E$ such that

$$
4 r_{i}=d\left(z_{i}, E\right)=\left|z_{i}-z^{\prime}\right| .
$$


Then for $z \in \gamma_{i} \cap B\left(z_{i}, r_{i}\right)$,

$$
d(z, E) \leq\left|z-z^{\prime}\right| \leq\left|z-z_{i}\right|+\left|z_{i}-z^{\prime}\right| \leq r_{i}+4 r_{i}=5 r_{i}
$$

and thus

$$
\int_{\gamma_{i}} d(z, E)^{-1} d s \geq \int_{\gamma_{i} \cap B\left(z_{i}, r_{i}\right)} d(z, E)^{-1} d s \geq r_{i} / 5 r_{i}=1 / 5
$$

Hence

$$
\int_{\gamma} d(z, E)^{-1} d s \geq \sum_{i=1}^{j-1} \int_{\gamma_{i}} d(z, E)^{-1} d s \geq(j-1) / 5
$$

and we obtain from $(3.4)$

$$
j \leq 5 M+6 \text {. }
$$

By the above construction $\partial G \cap \bar{B}\left(z_{j}, 2 r_{j}\right) \neq \emptyset$, hence there is $x_{0} \in \partial G \cap$ $\bar{B}^{n}\left(z_{j}, 2 r_{j}\right)$. Set $u=1-\omega$. Then $u$ is a solution of (1.3) in $G, 0 \leq u \leq 1$ and if we set $u(x)=1$ for $x \in \mathcal{C} G \cap B\left(z_{j}, 4 r_{j}\right)$, then $u$ is continuous in $B\left(z_{j}, 4 r_{j}\right)$. Consequently, $u$ is a continuous function in $B\left(x_{0}, 2 r_{j}\right)$ and a solution of (1.3) in $B\left(x_{0}, 2 r_{j}\right) \backslash \mathcal{C} G$. Let $F=\mathcal{C} G \cap \bar{B}\left(x_{0}, r_{j}\right)$. Thus Lemma 3.2 and the $p$-capacity density condition yield for $z \in B\left(x_{0}, r_{j}\right)$

$$
\begin{aligned}
u(z) & \geq c_{1} r_{j}^{(p-n) /(p-1)} \operatorname{cap}_{p}\left(F, B\left(x_{0}, 2 r_{j}\right)\right)^{1 /(p-1)} \\
& \geq c_{1} r_{j}^{(p-n) /(p-1)} c_{0} r_{j}^{(n-p) /(p-1)}=c_{1} c_{0}>0 .
\end{aligned}
$$

Hence for $z \in B\left(z_{j}, r_{j}\right)$ we have

$$
u(z) \geq c_{1} c_{0} .
$$

Set $B_{i}=B\left(z_{i}, r_{i}\right), i=1, \ldots, j$, and $u=1-\omega$. Then (3.6) and Lemma 3.3 yield

$$
c_{1} c_{0} \leq \inf _{B_{j}} u \leq \sup _{B_{j-1}} u \leq c_{2} \inf _{B_{j-1}} u \leq \cdots \leq c_{2}^{j-1} \inf _{B_{1}} u .
$$

Hence we obtain

$$
\omega(x)=1-u(x) \leq 1-\inf _{B_{1}} u \leq 1-c_{1} c_{0} c_{2}^{1-j}
$$

and (3.5) implies $\omega(x) \leq c<1$ where

$$
c=1-c_{1} c_{0} c_{2}^{-5 M-5} .
$$

This shows that $\omega(x) \leq c$ and the proof is complete. 
3.7. Remark. In the case $p=n$ it was shown in [GLM 2, 4.18 and 4.19] that if $E$ is a compcat set in the boundary of the unit ball $B$ and if the domain $R^{n} \backslash E$ is a uniform domain in the sense of $[\mathrm{MS}]$, then $\omega=\omega(E, B ; A)=0$. Note that $B$ satisfies a $p$-capacity density condition for all $p, 1<p \leq n$. Now Theorem 3.1 implies this result for all $A$. Hence it is easy to construct compact sets $E \subset \partial B$ whose Hausdorff-dimension is arbitrary close to $n-1$ and yet $\omega(E, B ; A)=0$ for all $A$.

On the other hand, since the neighborhoods $\mathcal{U}_{i}$ of Theorem 3.1 are at our disposal, it is easy to construct a compact set $E$ in $\partial B$ which satisfies (a) and (b) of 3.1 and yet $R^{n} \backslash E$ is not a uniform domain.

\section{Buried sets}

Let $G$ be a bounded domain in $R^{n}$. Write $C=\partial G$. For $r>0$ set

$$
C_{G}(r)=(C+B(r)) \cap G
$$

and for $c>0$ put

$$
C_{c}(r)=\left\{x \in C: d\left(x, \partial C_{G}(r) \cap G\right) \geq(1+c) r\right\} .
$$

Then $C_{c}(r)$ is a compact subset of $\partial G$.

A subset $E$ of $\partial G$ is said to be buried in $\partial G$ if there is a number $c>0$ and a sequence of positive numbers $r_{i} \rightarrow 0$ such that

$$
E \subset \cap_{i} C_{c}\left(r_{i}\right)
$$

It is easy to see that if $\partial G$ is a $C^{1}$-manifold, then no subset $E$ of $\partial G$ is buried in $\partial G$. Roughly speaking, a set $E$ is buried in $\partial G$ if there are numbers $r_{i} \searrow 0$ with the following property: If one stands at the distance $r_{i}$ from $\partial G$ in $G$, then the set $E$ is slightly further away than $\partial G$.

The following theorem generalizes $[\mathrm{H}$, Theorem $\mathrm{A}]$.

4.2. Theorem. Suppose that $G$ is a bounded domain which satisfies a $p$-capacity density condition. If a set $E$ is buried in $\partial G$, then $\omega(E, G ; A)=0$.

Proof. We may assume that $E$ is compact. Let $c>0$ and $\left(r_{i}\right)$ be such that $(4.1)$ holds. For each $i=1,2, \ldots$ write $\mathcal{U}_{i}=\partial G+B\left(r_{i}\right)$. Then $\mathcal{U}_{i}$ is a neighborhood of $\partial G$ and hence of $E$. Moreover, $\bigcap \mathcal{U}_{i} \cap G=\emptyset$. It remains to show that the condition (b) of Theorem 3.1 is satisfied.

To this end let $x \in \partial \mathcal{U}_{i} \cap G$. Then there exists $y \in \partial G$ such that

$$
|x-y|=d(x, \partial G)=r_{i} .
$$


Now

$$
d(x, E) \geq(1+c) r_{i}
$$

because in the opposite case

$$
(1+c) r_{i}>d(x, E) \geq d\left(x, C_{c}\left(r_{i}\right)\right) \geq(1+c) r_{i},
$$

a contradiction. Let $\gamma(t)=\left(t y+\left(r_{i}-t\right) x\right), t \in\left[0, r_{i}\right]$, be the straight line segment from $x$ to $y$. If we let $D=R^{n} \backslash E$, then

$$
\begin{aligned}
k_{D}(x, y) & \leq \int_{\gamma} d(z, E)^{-1} d s \leq \int_{0}^{r_{i}}\left[(1+c) r_{i}-t\right]^{-1} d t \\
& =\log \frac{1+c}{c}=M<\infty
\end{aligned}
$$

because by (4.3) for each $t \in\left[0, r_{i}\right]$

$$
d(\gamma(t), E) \geq(1+c) r_{i}-t .
$$

Hence the condition (b) of Theorem 3.1 is satisfied and $\omega(E, G ; A)=0$ follows from Theorem 3.1.

4.4. Remark. Simple examples show that there are bounded domains $G$ and sets $E$ buried in $\partial G$ such that $\partial G \backslash E$ is countable. Hence the $p$-capacity density condition in Theorem 4.2 cannot be completely removed. Slight modifications of the above example show that this condition cannot by replaced by the condition that $G$ is $A$-Dirichlet regular. 


\section{References}

[CFK] Caffarelli, L., E. Fabes, and C. Kenig: Completely singular elliptic harmonic measures. - Indiana Univ. Math. J. 30, 1981, 917-924.

[GP] Gehring, F.W., and B. Palka: Quasiconformally homogeneous domains. - J. Analyse Math. 30, 1976, 172-199.

[GLM1] Granlund, S., P. Lindqvist, and O. Martio: Conformally invariant variational integrals. - Trans. Amer. Math. Soc. 277, 1983, 43-73.

[GLM2] Granlund, S., P. Lindqvist, and O. Martio: $F$-harmonic measure in space. - Ann. Acad. Sci. Fenn. Ser. A I Math. 7, 1982, 233-247.

[H] Heinonen, J.: Boundary accessibility and elliptic harmonic measures. - Complex Variables $10,1988,273-282$.

[HK] Heinonen, J., and T. KilpeläInEN: $A$-superharmonic functions and supersolutions of degenerate elliptic equations. - Ark. Mat. 26, 1988, 87-105.

[HM] Heinonen, J., and O. Martio: Estimates for $F$-harmonic measures and $\varnothing$ ksendal's theorem for quasiconformal mappings. - Indiana Univ. Math. J. 36, 1987, 659-683.

[M] Martio, O.: $F$-harmonic measures, quasihyperbolic distance and Milloux's problem. Ann. Acad. Sci. Fenn. Ser. A I Math. 12, 1987, 151-162.

[MS] Martio, O., and J. Sarvas: Injectivity theorems in plane and space. - Ann. Acad. Sci. Fenn. Ser. A I Math. 4, 1978/1979, 383-401.

[Maz] MAZ'YA, V.G.: On the continuity on a boundary point of solutions of quasi-linear elliptic equations. - Vestnik Leningrad Univ. Mat. 13, 1970, 42-55 (Russian).

[Ø] ØKSENDAL, B.: Dirichlet forms, quasiregular functions and Brownian motion. - Invent. Math. 91, 1988, 273-297.

[S] SERrin, J.: Local behavior of solutions of quasilinear equations. - Acta Math. 111, 1964, 247-302.

University of Jyväskylä

Department of Mathematics

Seminaarinkatu 15

SF-40100 Jyväskylä

Finland

Received 10 October 1987 